\title{
Sequence analysis for detection of first-line drug resistance in Mycobacterium tuberculosis strains from a high-incidence setting
}

Silke Feuerriegel ${ }^{1 *}$, Barbara Oberhauser ${ }^{2}$, Abu Garawani George ${ }^{3}$, Foday Dafae ${ }^{4}$, Elvira Richter ${ }^{5}$, Sabine Rüsch-Gerdes ${ }^{5}$ and Stefan Niemann ${ }^{1}$

\begin{abstract}
Background: Drug resistance displays a problem for the therapy of Mycobacterium tuberculosis infections. For molecular resistance testing, it is essential to have precise knowledge on genomic variations involved in resistance development. However, data from high-incidence settings are only sparely available. Therefore we performed a systematic approach and analyzed a total of $97 \mathrm{M}$. tuberculosis strains from previously treated patients in Sierra Leone for mutations in $k a t G, r p o B$, rrs, rps $L$, gidB, embB, pncA and where applicable in inhA and ahpC. Of the strains investigated 50 were either mono- or poly-resistant to isoniazid, rifampin, streptomycin, ethambutol and pyrazinamide or MDR and 47 fully susceptible strains served as controls.

Results: The majority of isoniazid and rifampin resistant strains had mutations in katG315 (71.9\%) and rpoB531 (50\%). However, rpoB mutations in codons 511, 516 and 533 were also detected in five rifampin susceptible strains. MIC determinations revealed low-level rifampin resistance for those strains. Thus, the sensitivity and specificity of sequencing of $\mathrm{kat} \mathrm{G}$ for detection of drug resistance were $86.7 \%$ and $100 \%$ and for sequencing of rpoB $100 \%$ and $93.8 \%$, respectively.

Strikingly, none of the streptomycin resistant strains had mutations in rrs, but $47.5 \%$ harboured mutations in rpsL. Further changes were detected in gidB. Among ethambutol resistant strains $46.7 \%$ had mutations at embB306. Pyrazinamide resistant strains displayed a variety of mutations throughout pncA. The specificities of sequencing of rpsL, embB and pncA for resistance detection were high (96-100\%), whereas sensitivities were lower (48.8\%, 73.3\%, 70\%).

Conclusions: Our study reveals a good correlation between data from molecular and phenotypic resistance testing in this high-incidence setting. However, the fact that particular mutations in rpoB are not linked to high-level resistance is challenging and demonstrates that careful interpretation of molecular resistance assays is mandatory. In addition, certain variations, especially in gidB, appear to be phylogenetically informative polymorphisms rather than markers for drug resistance.
\end{abstract}

\section{Background}

With more than 9 million new tuberculosis (TB) cases and about 1.7 million deaths in 2009 [1] TB remains one of the most serious infectious diseases worldwide. Treatment and control of TB is further complicated by the emergence of drug resistant and even multi drug resistant (MDR) strains [resistance to at least isoniazid (INH) and rifampin (RIF)] [2]. Among high-incidence settings, Sub-Saharan Africa is eminently affected with two

\footnotetext{
* Correspondence: sfeuerriegel@fz-borstel.de

${ }^{1}$ Molecular Mycobacteriology, Research Center Borstel, Borstel, Germany

Full list of author information is available at the end of the article
}

million new TB cases per year [3]. This study focuses on Sierra Leone, a high burden country with an annual TB incidence rate of 574 per 100.000 people and an annual mortality rate of 149 per 100.000 people. Treatment options are further hampered by the fact that $23 \%$ among previously treated TB patients in Sierra Leone suffer from an MDR M. tuberculosis strain [4].

Rapid detection of resistance is the key task to ensure an effective treatment of patients and also to avoid further spread of resistant M. tuberculosis strains. Molecular assays that detect the genetic variants that mediate resistance constitute a rapid alternative to conventional

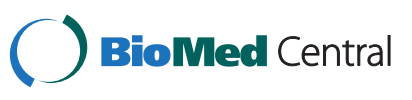


drug susceptibility testing (DST) and may even be performed directly on clinical specimens without culture [5,6]. Therefore it is essential to elucidate the genetic basis of clinical resistance and to correlate phenotypic and molecular resistance data.

Resistance to INH is predominantly mediated by one mutation in the kat G gene at codon 315 which results in the complete or partial loss of catalase-peroxidase activity [7]. Further mutations in the promoter regions of inhA $[8]$ and $\operatorname{ahpC}[9,10]$ are associated with INH resistance. Mutations responsible for RIF resistance are primarily located in the so-called rifampin resistance determining region (RRDR; codon 507-533 according to E. coli numbering system) of the rpoB gene which encodes the beta subunit of the RNA polymerase [11]. Resistance to streptomycin (SM) is mediated by mutations in different genes. Polymorphisms in rrs and rpsL, coding for $16 \mathrm{~S}$ rRNA and the ribosomal protein S12, respectively, are mainly responsible for high-level resistance [12]. Recently, the gidB gene, which encodes a 7-methylguanosine methyltransferase specific for $16 \mathrm{~S}$ rRNA, has additionally been associated with SM resistance [13]. Resistance to ethambutol (EMB) is primarily mediated by mutations in the $e m b \mathrm{~B}$ gene, coding for an arabinosyltransferase participating in mycobacterial cell wall synthesis, with codon 306 being most frequently affected [14]. Furthermore, mutations in other parts of $e m b \mathrm{~B}$ (e.g. codon 406) [15] and upstream of embA $[15,16]$ and in $e m b C[16,17]$ are also involved in EMB resistance. Resistance to pyrazinamide (PZA) is known to be mediated by mutations occurring throughout the
pncA gene, encoding a pyrazinamidase [18]. Resistant strains lack pyrazinamidase activity which is essential for pro drug activation.

Since the frequency and combination of resistance mutations differs depending on the geographical setting in which the specific isolate is found $[19,20]$, it is important to analyze Mycobacterium tuberculosis complex (MTBC) strains from different regions and to determine putative setting specific molecular markers. However, up to now data about the accuracy of molecular diagnostic methods in high-incidence settings, and especially in West Africa, is only sparely available. Therefore we carried out a population based study, involving MTBC strains from Sierra Leone, to determine the genetic basis of first line drug resistance and to compare results from molecular and conventional drug susceptibility testing.

\section{Methods}

Mycobacterial strains and growth conditions

A total of 97 MTBC strains isolated from previously treated patients in Sierra Leone were included in this study. All smear positive cases registered for retreatment (failure after at least 5 months, relapses or treatment after interruption) between March 2003 and June 2004 in the Western Area and Kenema districts in Sierra Leone were recruited. From the strains analyzed 50 were resistant to at least one of the following drugs INH, RIF, SM, EMB and PZA and 47 strains were fully susceptible (see Figure 1). From the panel of strains analyzed, 74 were $M$. tuberculosis and 23 were $M$. africanum strains. Primary isolation and cultivation was done

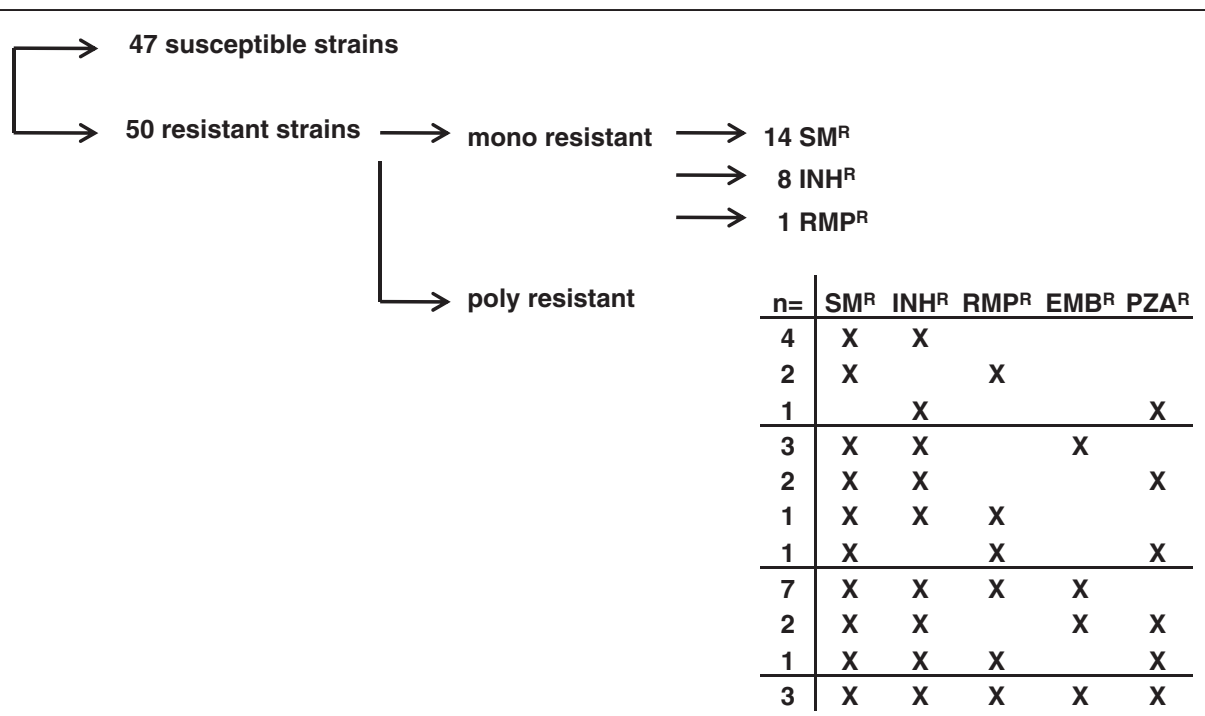

Figure 1 Overview of the antibiotic resistance profiles of the strains analyzed. A total of 97 M. tuberculosis and M. africanum strains from smear positive, previously treated patients from Sierra Leone was included in this study. Samples were collected in 2003 and 2004 in the Western Area and Kenema districts. Of the strains analyzed 74 were M. tuberculosis and 23 were M. africanum strains. Abbreviations: INH, isoniazid; RIF, rifampin; SM, streptomycin; EMB, ethambutol; PZA, pyrazinamide; R, resistance. 
at the Supranational Reference Laboratory in Borstel as described previously [21].

\section{Drug susceptibility testing}

Drug susceptibility testing (DST) to first-line drugs INH $(0.25$ and $1.0 \mu \mathrm{g} / \mathrm{ml})$, RIF (20.0 and $40.0 \mu \mathrm{g} / \mathrm{ml})$, SM (4.0 and $8.0 \mu \mathrm{g} / \mathrm{ml})$ and EMB (1.0 and $2.0 \mu \mathrm{g} / \mathrm{ml})$ was performed in Borstel by using the proportion method on Löwenstein-Jensen (LJ) medium. In case of insufficient growth on LJ media, DST was done by applying the modified proportion method in the BACTEC 460 TB system according to the manufacturer's instructions (Becton-Dickinson). Drug susceptibility testing to PZA $(100 \mu \mathrm{g} / \mathrm{ml})$ was performed by using the BACTEC ${ }^{\mathrm{mm}}$ Pyrazinamide (PZA) Drug Kit in the BACTEC 460 TB system according to the manufacturer's instructions. Determination of minimal inhibitory concentrations (MICs) was done by applying the modified proportion method in the MGIT 960 TB system (test concentrations were $1.0,0.5,0.25,0.125$ and $0.063 \mu \mathrm{g} / \mathrm{ml}$ for RIF and SM, 100.0, 50.0, 25.0, 12.5 and $6.3 \mu \mathrm{g} / \mathrm{ml}$ for PZA).

\section{DNA Isolation, PCR and sequencing}

DNA was isolated as described elsewhere [22] and amplified using the primers and conditions listed in Additional file 1 . The PCR products were sequenced using an ABI 3130xl Genetic Analyzer (Applied Biosystems, CA, US) and the ABI BigDye Terminator kit v.1.1 according to the manufacturer's instructions. The sequence data was analyzed using DNASTAR Lasergene version 8.0, with $M$. tuberculosis H37Rv DNA as reference sequence.

All strains were sequenced in the predominant resistance determining regions (RDR) of katG (codon 315), rpoB (codon 507-533 according to E. coli numbering), rrs (nt. 1401-1402), rpsL (complete gene), gid $\mathrm{B}$ (complete gene), embB (codon 306) and pncA (complete gene). Strains resistant to the respective drug but not carrying a mutation in the RDR were sequenced in the complete gene. Strains resistant to INH with no mutation in $k a t \mathrm{G}$ were also sequenced in the promoter regions of inhA and $a h p C$. Similarly, we extended our sequencing effort to $e m b C$ and $e m b \mathrm{~A}$ for EMB resistant strains without any mutations in $e m b B$.

\section{Results}

In this study a total of 97 MTBC strains with known resistance patterns to first-line drugs from Sierra Leone (see Figure 1) were sequenced in genes previously described to be involved in resistance development.

The population structure of the strains was analyzed in a previous study [21]. Briefly, the 74M. tuberculosis and $23 \mathrm{M}$. africanum strains belonged to eleven different genotypes. The population diversity was high with two
M. africanum lineages (West African $\mathrm{I}, \mathrm{n}=6$; West African II, $\mathrm{n}=17$ ) and nine M. tuberculosis lineages (Haarlem, $\mathrm{n}=14 ;$ LAM, $\mathrm{n}=15$; EAI, $\mathrm{n}=4$; Beijing, $\mathrm{n}=4$; S-type, $\mathrm{n}=4$; X-type, $\mathrm{n}=1$; Cameroon, $\mathrm{n}=4$; Sierra Leone I, $\mathrm{n}=7$; Sierra Leone II, $\mathrm{n}=10$ ). To determine if certain mutations appear genotype specific, the occurrence of identified polymorphisms was correlated with the genotype of the respective strain. However, all mutations detected by sequencing analysis were found independently from their phylogenetic background (data not shown). A detailed summary of the sequencing data is provided in Table 1 and in Figure 2 (a-e).

Among the INH resistant strains 71.9\% (23/32) carried a mutation in katG at codon 315 . Out of these, 21 displayed a mutation in katG only, while two strains showed mutations at katG315 with additional mutations at codon 291 and codon 471, respectively. One strain each carried a mutation at codon 300, codon 302 and codon 329. Two resistant strains displayed a mutation at codon 463, which is a phylogenetic SNP [23] and was therefore excluded from further analysis. Four of the INH resistant strains had no mutation in katG. However, sequence analysis of the intergenic regions of inh A and $a h p C$ revealed polymorphisms in those areas. Two strains carried a mutation in inhA at position -15 and one strain in $a h p C$ at -57 . All of the $65 \mathrm{INH}$ susceptible strains lacked mutations in katG. Thus for detection of INH resistance, sequence analyses of $k a t \mathrm{G}$ had a sensitivity and specificity of $86.7 \%$ and $100 \%$, in the strains analyzed.

Among RIF resistant strains, 50\% (8/16) carried a mutation in rpoB at codon 531. The second most frequent mutation was found at codon 526 (37.5\%). One RIF resistant strain each showed a mutation at codon 481 and at codon 533, respectively. Out of 81 RIF susceptible strains 76 did not have any mutation in rpoB. The remaining five susceptible strains displayed mutations at codons $511(\mathrm{n}=1), 516(\mathrm{n}=3)$ and $533(\mathrm{n}=1)$, respectively. Sequence analysis and drug susceptibility testing has been repeated for those five strains, confirming results of the first analyses. Determination of MICs revealed low-level RIF resistance $(0.25-1.0 \mu \mathrm{g} / \mathrm{ml})$ for those strains (see Table 2). Given that the strains showing low-level RIF resistance are assessed as susceptible by using standard DST, sequence analyses of rpoB had a sensitivity and specificity of $100 \%$ and $93.8 \%$ for detection of RIF resistance, in the strains analyzed.

To investigate the genetic basis of SM resistance, all strains were first sequenced in the rrs gene. As none of the resistant strains displayed a mutation in this gene, sequence analysis of $r p s \mathrm{~L}$ was performed. Among all SM resistant strains $27.5 \%(11 / 40)$ carried a mutation in rpsL at codon 43 and $20 \%(8 / 40)$ showed a polymorphism at codon 88 . The remainder of the phenotypically resistant 
Table 1 Mutations detected in all strains analyzed

\begin{tabular}{|c|c|c|c|}
\hline Gene & Mutation & $\begin{array}{l}\text { Strains phenotypically } \\
\text { susceptible to INH ( } n=)\end{array}$ & $\begin{array}{l}\text { Strains phenotypically } \\
\text { resistant to INH }(n=)\end{array}$ \\
\hline \multirow[t]{9}{*}{$\overline{k a t G}$} & wild type & 65 & $4^{(1)}$ \\
\hline & Ala291Thr (gct/act); Ser315Thr (agc/acc) & & 1 \\
\hline & Trp300Cys (tgg/tgc) & & 1 \\
\hline & Thr302Arg (acg/agg) & & 1 \\
\hline & Ser315Thr (acg/acc) & & 21 \\
\hline & Asp329 (gac/ggac frameshift) & & 1 \\
\hline & Arg463Leu $(\mathrm{cgg} / \mathrm{ctg})^{(2)}$ & & 2 \\
\hline & Gln471Arg (cag/cgg); Ser315Thr (agc/acc) & & 1 \\
\hline & & $\begin{array}{l}\text { Strains phenotypically } \\
\text { susceptible to RIF ( } n=)\end{array}$ & $\begin{array}{l}\text { Strains phenotypically } \\
\text { resistant to RIF }(n=)\end{array}$ \\
\hline \multirow[t]{9}{*}{ rpoB } & wild type & 76 & \\
\hline & Thr481Ala (acc/gcc) ${ }^{(3)}$ & & 1 \\
\hline & Leu511Pro $(\mathrm{ctg} / \mathrm{ccg})^{(4)}$ & 1 & \\
\hline & Asp516Tyr (gac/tac) ${ }^{(4)}$ & 3 & \\
\hline & His526Tyr (cac/tac) & & 3 \\
\hline & His526Arg (cac/cgc) & & 3 \\
\hline & Ser531 Leu (tcg/ttg) & & 8 \\
\hline & Leu533Pro (ctg/ccg) ${ }^{(4)}$ & 1 & 1 \\
\hline & & $\begin{array}{l}\text { Strains phenotypically } \\
\text { susceptible to SM ( } n=)\end{array}$ & $\begin{array}{l}\text { Strains phenotypically } \\
\text { resistant to } S M(n=)\end{array}$ \\
\hline \multirow[t]{3}{*}{$r p s L$} & wild type & 56 & 21 \\
\hline & Lys43Arg (aag/agg) & & 11 \\
\hline & Lys88Arg (aag/agg) & 1 & 8 \\
\hline \multirow[t]{20}{*}{ gidB } & wild type & 26 (2 with mutations in $r r s / r p s L)$ & $\begin{array}{l}15 \text { (10 with mutations } \\
\text { in } r p s \mathrm{~L})\end{array}$ \\
\hline & Leu16Arg (ctt/cgt) & 4 & 2 \\
\hline & $\begin{array}{l}\text { Leu16Arg (ctt/cgt); Gln127stop (caa/taa); } \\
\text { Ala161Ala (gcc/gcg) }\end{array}$ & 1 & \\
\hline & Leu16Arg (ctt/cgt); Ala161Ala (gcc/gcg) & 2 & 1 (Lys43Arg in rpsL) \\
\hline & Leu16Arg (ctt/cgt); Ala200Glu (gcg/gag) & & 2 \\
\hline & Leu16Arg (ctt/cgt); Ala205Ala (gca/gcg) & & 1 (Lys43Arg in rpsL) \\
\hline & Gly34Ala (ggg/gcg) & & 1 \\
\hline & Pro38 (ccc/cc frameshift) & & 1 (Lys88Arg in rpsL) \\
\hline & Val65Gly (gtc/ggc) & & 1 \\
\hline & Gly69Asp (ggt/gat) & 1 & 2 \\
\hline & Gly71Arg (gga/aga) & & 1 \\
\hline & Val88Ala (gta/gca) & & 1 \\
\hline & Leu91Pro (cta/cca) & & 1 \\
\hline & Gly92Asp (gaa/gac) & 1 & \\
\hline & Gly92Asp (gaa/gac); Ala205Ala (gca/gcg) & & 3 (Lys43Arg in rpsL) \\
\hline & Ser100Phe (tct/ttt) & & 1 \\
\hline & Val1 10Val (gtg/gtt); Ala205Ala (gca/gcg) & 4 & \\
\hline & Ala138Val (gcg/gtg) & & 2 \\
\hline & Ala200Glu (gcg/gag) + Ala205Ala (gca/gcg) & & 1 \\
\hline & Ala205Ala (gca/gcg) & 18 & 4 (3 with mutations in rps $\mathrm{L}$ \\
\hline
\end{tabular}


Table 1 Mutations detected in all strains analyzed (Continued)

\begin{tabular}{|c|c|c|c|}
\hline & & $\begin{array}{l}\text { Strains phenotypically } \\
\text { susceptible to EMB }(n=)\end{array}$ & $\begin{array}{l}\text { Strains phenotypically } \\
\text { resistant to EMB }(n=)\end{array}$ \\
\hline \multirow[t]{7}{*}{$\overline{e m b B}$} & wild type & 82 & $4^{(5)}$ \\
\hline & Met306lle (atg/ata) & & 6 \\
\hline & Met306Val (atg/gtg) & & 1 \\
\hline & Trp332Arg (tgg/cgg) & & 1 \\
\hline & Gln497Lys (cag/aag) ${ }^{(3)}$ & & 1 \\
\hline & Gln1002Arg (cag/cgc) $)^{(3)}$ & & 2 \\
\hline & & $\begin{array}{l}\text { Strains phenotypically } \\
\text { susceptible to PZA }(n=)\end{array}$ & $\begin{array}{l}\text { Strains phenotypically } \\
\text { resistant to PZA }(n=)\end{array}$ \\
\hline \multirow[t]{10}{*}{ pncA } & wild type & 84 & 3 \\
\hline & $-11(\mathrm{a} / \mathrm{g})$ & & 1 \\
\hline & Ile5 (atc/atcc frameshift) & & 1 \\
\hline & Thr47Ala (acc/gcc) & 2 & \\
\hline & Tyr64 (tat/tata frameshift) & & 1 \\
\hline & Lys96Glu (aag/gag) & 1 & \\
\hline & $\begin{array}{l}\text { Gln141 (cag/cag acggcgccag (insertion of } \\
10 \mathrm{bp} \rightarrow \text { frameshift) }\end{array}$ & & 1 \\
\hline & Ala146Glu (gcg/gag) & & 1 \\
\hline & Gly162Asp (ggt/gat) & & 1 \\
\hline & Leu172Pro (ctg/ccg) & & 1 \\
\hline
\end{tabular}

Sequence analysis and drug susceptibility testing has been repeated for all strains showing discrepant results.

Abbreviations: INH, isoniazid; RIF, rifampin; SM, streptomycin; EMB, ethambutol; PZA, pyrazinamide.

${ }^{(1)}$ Those strains were additionally sequenced in the promoter region of inhA and ahpC. Two of them carried a mutation in inhA at position $-15(C \rightarrow \mathrm{T})$ and one in $\operatorname{ahpC}$ at $-57(\mathrm{C} \rightarrow \mathrm{T})$.

${ }^{(2)}$ Arg463Leu is a phylogenetic SNP [23] and was excluded for further analysis.

${ }^{(3)}$ These mutations were identified by sequence analysis of the complete gene.

${ }^{(4)}$ These mutations confer low-level RIF resistance.

${ }^{(5)}$ These strains were sequenced in the complete embCAB operon. One strain carried a mutation in embC [Val981 Leu (gtg/ctg)].

strains $(\mathrm{n}=21)$ did not carry a mutation in rpsL. Among all SM susceptible strains $(\mathrm{n}=57)$, one had the codon 88 mutation in rpsL as well (confirmed when retested). Determination of SM MIC showed no elevated MIC for the respective strain compared to the $\mathrm{H} 37 \mathrm{Rv}$ control (see Table 2). Taken together, these data resulted in a sensitivity and specificity of the DNA sequencing of rpsL for detection of SM resistance of $48.8 \%$ and $98.2 \%$, respectively.

Additionally all strains were sequenced in gidB. In this very polymorphic gene 16 different mutations have been found, which occurred alone or in combination (see Table 1). Noticeable is the high number of phylogenetic polymorphisms. The Leu16Arg (ctt/cgt) mutation was exclusively found in strains of the LAM genotype $(n=12)$. All strains belonging to the WA1, WA2 and Beijing genotypes displayed the Ala205Ala (gca/gcg) mutation $(\mathrm{n}=27)$ and in all EAI strains a combination of the Val110Val (gtg/gtt) and Ala205Ala (gca/gcg) mutations was detected $(n=4)$. The role of mutations in gidB for resistance to SM needs to be further investigated.
Among all EMB resistant isolates 46.7\% (7/15) carried a mutation in $e m b B$ at codon 306 . One EMB resistant strain was found to have a mutation at codon 332, one at codon 497 and two strains carried a polymorphism at codon 1002. In four EMB resistant isolates no mutation in $e m b \mathrm{~B}$ was detected. Sequence analyses of $e m b C$ and $e m b \mathrm{~A}$ revealed a mutation in embC [Val981Leu (gtg/ ctg)] in one strain. All EMB susceptible strains $(n=82)$ had a wild-type $e m b \mathrm{~B}$ sequence. Thus for detection of EMB resistance, sequence analyses of $e m b \mathrm{~B}$ had a sensitivity and specificity of $73.3 \%$ and $100.0 \%$, in the strains analyzed.

PZA resistant isolates showed a wide variety of changes, distributed throughout the entire length of the pncA gene, including its promoter. Single nucleotide polymorphisms (SNPs) occurred in one strain each at position $-11 \mathrm{bp}$, at codons 146, 162 and 172. In addition, insertions of single nucleotides leading to open reading frameshifts were detected at codons 5 and 64; an insertion of $10 \mathrm{bp}$ after codon 141 led to PZA resistance in one strain. In three resistant isolates no mutation in pncA was determined. Among all PZA susceptible strains 

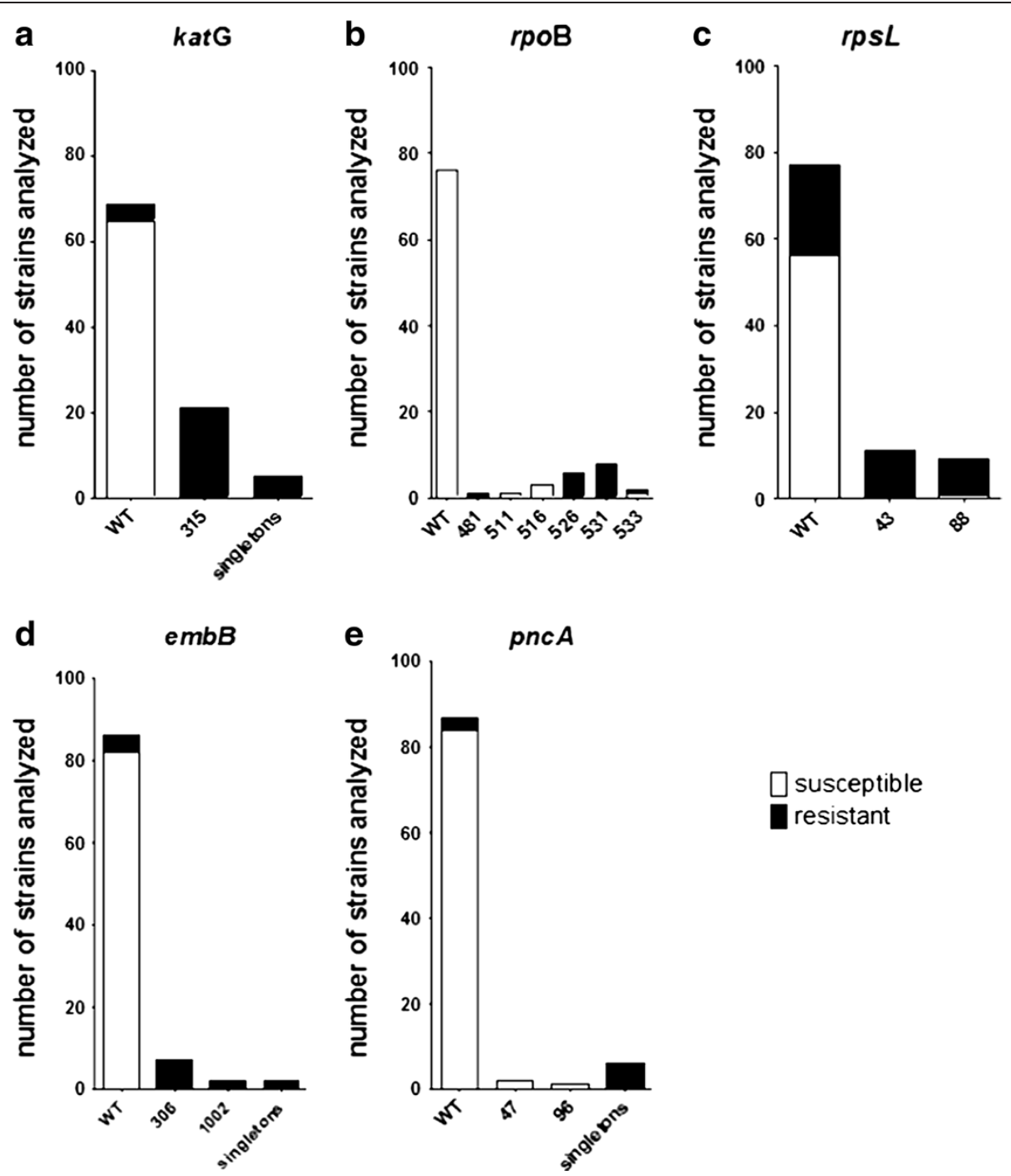

Figure 2 Overview of mutations detected in all strains analyzed in a) kat $\mathrm{G}, \mathrm{b}) \mathrm{rpoB}, \mathrm{c}) \mathrm{rps} \mathrm{L}, \mathrm{d})$ emb $\mathrm{B}$ and e) pncA. The height of the columns represent the number of strains analyzed. On the $x$-axis the different mutated codons are shown. The white areas of the columns represent the fraction of susceptible strains, whereas the black areas correspond to the number of resistant strains. Abbreviations: WT, wild type; singletons, various codons that are affected in one strain only.

$(\mathrm{n}=87), 84$ displayed the wild type sequence, whereas in three PZA susceptible strains mutations were detected at codon $47(n=2)$ and at codon $96(n=1)$, respectively. Sequence analysis and drug susceptibility testing has been repeated for strains showing discrepant results, however leading to unaltered findings. Determination of PZAMICs (see Table 2) revealed slightly elevated MICs for the strains carrying the mutation at codon $47(25.0 \mu \mathrm{g} /$ $\mathrm{ml}$ ) compared to the $\mathrm{H} 37 \mathrm{Rv}$ control, but an unaltered MIC for the strain carrying the polymorphism at codon 96. These data resulted in a sensitivity and specificity of the DNA sequencing of $p n c$ A for detection of PZA resistance of $70 \%$ and $96.6 \%$, respectively.

A summary of all sequencing, DST, MIC and genotyping data is provided in Additional file 2.

\section{Discussion}

In this study we carried out an in depth investigation of molecular resistance mechanisms by correlating particular genomic variants with phenotypic resistance in clinical isolates from a high-incidence setting in West Africa. For INH and RIF there is a close correlation between data from molecular and phenotypic resistance testing for resistance determination in the strains analyzed. Sensitivity and specificity of sequencing of kat $\mathrm{G}$ for detection of drug resistance were $86.7 \%$ and $100 \%$ and for sequencing of $r p o B \quad 100 \%$ and 93.8\%, respectively.

Overall, the correlation between molecular and phenotypic resistance testing for the determination of $\mathrm{SM}, \mathrm{EMB}$ and PZA resistance was lower. Although 
Table 2 Determination of minimal inhibitory concentrations (MICs) of potential low-level resistant strains (to RIF, SM, PZA)

\begin{tabular}{|c|c|c|}
\hline strain & mutation & RIF MIC $[\mu \mathrm{g} / \mathrm{ml}]$ \\
\hline $4518 / 03$ & rpoB Asp516Tyr (gac/tac) & 0.5 \\
\hline $5472 / 03$ & rpoB Leu533Pro (ctg/ccg) & 1.0 \\
\hline $10011 / 03$ & rpoB Asp516Tyr (gac/tac) & 0.5 \\
\hline $3736 / 04$ & rpoB Leu511Pro (ctg/ccg) & 0.5 \\
\hline $6467 / 04$ & rpoB Asp516Tyr (gac/tac) & 0.25 \\
\hline H37Rv control & wild type & 0.25 \\
\hline strain & mutation & SM MIC $[\mu \mathrm{g} / \mathrm{ml}]$ \\
\hline $6463 / 04$ & rpsL Lys88Arg (aag/agg) & 0.5 \\
\hline H37Rv control & wild type & 0.5 \\
\hline strain & mutation & PZA MIC $[\mu \mathrm{g} / \mathrm{ml}]$ \\
\hline $4724 / 03$ & pncA Thr47Ala (acc/gcc) & 25.0 \\
\hline $4730 / 03$ & pncA Thr47Ala (acc/gcc) & 25.0 \\
\hline $6467 / 04$ & pncA Lys96Glu (aag/gag) & 12.5 \\
\hline H37Rv control & wild type & 12.5 \\
\hline
\end{tabular}

specificities of sequencing of $r p s \mathrm{~L}, e m b \mathrm{~B}$ and $p n c \mathrm{~A}$ were high (96-100\%), sensitivities were lower (48-73\%) due to so far unknown resistance mechanisms.

However, while our results in principle support molecular resistance testing, the finding that especially in rpo $\mathrm{B}$ and also in pncA particular mutations are not linked to high-level resistance is challenging and demonstrates that careful interpretation of molecular resistance assays is mandatory. Therefore, studies targeting new resistance mechanisms should include valid phenotypic resistance data and, to our opinion, a comprehensive database on genetic variations in resistance genes and the correlation with phenotypic resistance is necessary. Furthermore, the level of resistance mediated by particular mutations and the clinical consequences need to be thoroughly investigated. In addition, especially variations in gid $\mathrm{B}$ appear to be phylogenetically restricted rather than being involved in drug resistance development.

In our study the most frequent mutation among $\mathrm{INH}$ resistant strains has been detected in kat $\mathrm{G}$ at codon 315 . This SNP has been observed in numerous prior studies $[24,25]$ and has clearly been correlated with INH resistance by loss of catalase activity. In two strains, in addition to variations at katG315, mutations at codon 291 and 471 were detected. However neither mutation has been described in the literature before and the katG315 mutation therefore represents the likely mechanism for INH resistance in these strains. The mutation at codon 300 observed in one strain in our study has been previously reported by Richardson and co-workers [26], where loss of this mutation has resulted in reversion of INH resistance in a previously drug resistant strain. The mutation at codon 302 as well as the insertion at codon 329 has not been described previously. Since they are restricted to INH resistant strains in our highly diverse MTBC collection, they represent potential new INH resistance mechanisms. Experimental evidence is required to validate this hypothesis.

Of the four INH resistant strains that displayed a wildtype kat $\mathrm{G}$ sequence, three had mutations in the promoter regions of inh $\mathrm{A}$ and $a h p \mathrm{C}$. The inhA mutation has previously been described in the literature [24] as being the most common variation in the inhA promoter region related to INH resistance. Mutations in ahpC have been found before, however to our knowledge not at this position. In one of the resistant strains no mutation was found in neither the complete katG gene nor in inh $\mathrm{A}$ or in $a h p C$. This result suggests a so far unknown resistance mechanism as being responsible for INH resistance of this strain.

Mutations in rpo B at codons 526 and 531 occur most frequently in the RIF resistant strains analyzed. Those SNPs are located in the RRDR and are well known for mediating resistance $[27,28]$. The mutation at codon 481, which only occurs in one RIF resistant isolate, has to our knowledge not been described previously.

The mutations at codon 511 (Leu $\rightarrow$ Pro), 516 (Asp $\rightarrow$ Tyr) and 533 (Leu $\rightarrow$ Pro) conferred low-level resistance in agreement with previous studies [29,30]. It has been shown that various substitutions in the same codon lead to different levels of resistance. For example mutations at codon 516 can confer either low- or high-level resistance depending on the amino acid change [30]. Furthermore, the phenomenon of RIF low-level resistance has only recently been described in a work by Van Deun and colleagues [31], where mutations at codon 511, 516 and 533 have been found in strains tested susceptible by the radiometric Bactec 460 TB and Bactec 960 MGIT methods. Our data confirm the existence of low-level RIF resistance mediated by specific mutations in rpoB that is not detected by standard drug susceptibility testing methods. However, MIC values, especially for the mutations at codon 516 and 533, are even lower $(0.5-1.0 \mu \mathrm{g} /$ $\mathrm{ml}$ ) than have been described in the literature. This fact may be due to the presence of further mutations in the operon or in other regions of the genome.

In a recent study [32] the therapeutic challenge of low-level RIF resistance has been addressed and may, according to the authors, be overcome by the application of higher RIF doses $(20 \mathrm{mg} / \mathrm{kg})$ in treatment regimens. However, the clinical relevance and interpretation of these data is still not fully understood and needs further investigation in animal treatment models or clinical trials.

Despite these discordant findings, we found a good correlation between the results from molecular and phenotypic testing for INH and RIF, as has been 
observed in another study [33]. In fact, the strains analyzed in this study predominantly harbour well described mutations which allows for the application of standard sequencing protocols or commercial line probe assays.

The analysis of SM resistance mechanisms revealed an interesting observation. None of the SM resistant strains carried a mutation in the rrs gene, although those mutations have been described as main resistance mechanisms that confer high-level SM resistance [12]. Instead, the SM resistant strains in our study population carry mutations in rpsL at codon 43 or 88 or at various codons in the gid $\mathrm{B}$ gene. The two mutations in rps $\mathrm{L}$ have been described previously to confer high-level SM resistance [28,34]. Polymorphisms in gidB were reported to confer a lower level of SM resistance [13]. However, due to a number of phylogenetic polymorphisms in $g i d \mathrm{~B}$, cautious interpretation of sequencing data is mandatory. Leu16Arg (ctt/cgt) has been described previously as phylogenetic marker for the LAM genotype [35], which could be confirmed in this study. Additionally, a synonymous SNP at codon Ala205Ala (gca/gcg) was identified as being specific for the WA1, WA2 and Beijing genotypes, as well as a combination of Ala205Ala (gca/ $\mathrm{gcg}$ ) and Val110Val (gtg/gtt) was determined as phylogenetically specific for strains belonging to the EAI genotype. These mutations in gidB occurred both in SM susceptible and resistant strains, affirming their role as phylogentic SNPs rather than markers for SM resistance. Polymorphisms in gidB probably playing a role in SM resistance, as they occur exclusively in SM resistant strains and do not coincide with mutations in rpsL, were detected throughout the complete gene (codons 34, 65, $71,88,91,100,138,200)$. However, the actual importance of these SNPs for SM resistance needs to be investigated in further studies.

Reasons for the absence of $r$ rs mutations in the strains analyzed and the shift to mutations in rpsL and $g i d \mathrm{~B}$ are mainly unclear, but are in line with previous studies reporting a disequilibrium in the distribution of resistance conferring mutations in different geographical areas or among strains of different genotypes [36-38]. Our findings confirm that the performance of molecular assays that only target particular mutations can be influenced by the differential prevalence of particular mutations in a given geographical area. Therefore, strain diversity needs to be considered and investigated before the new implementation of molecular assays in a study region.

Among EMB resistant isolates, the most frequent mutation affected codon 306 (Met/lle) of the embB gene. This mutation has been described in various studies as the main mutation mediating resistance to EMB [14,39]. The mutation at codon 497 has also been previously described in clinical isolates [40]. Moreover, both mutations have been shown to confer resistance by transfer in a wild type genetic background using allelic exchange experiments [41]. However, the authors conclude that single mutations only modestly increase resistance to EMB and additional so far unknown mutations are necessary to cause high-level resistance.

The mutations at codon 332 and 1002 determined here have not been described before. The impact of these changes has to be investigated in further studies. In four resistant strains no mutations were detected in the $e m b \mathrm{~B}$ region analyzed. Additional analysis of the $e m b C$ and $e m b \mathrm{~A}$ genes revealed a mutation in $e m b C$ [Val981Leu (gtg/ctg)] in one strain, belonging to the Haarlem genotype. However, this mutation has been described earlier as being specific for the Haarlem genotype and is not associated with resistance to EMB [16]. As mentioned above, other so far unknown resistance mediating mechanisms are probably responsible for the resistance phenotype in these four strains.

Mutations or insertions in the pncA gene are known to mediate PZA resistance [42,43], as observed in our study. No hotspot region has been determined, since polymorphisms occur throughout the complete gene. However, according to our data some specific mutations do obviously not mediate resistance that is detectable by applying standard critical concentrations. In the panel of strains analyzed, two susceptible strains carry a SNP at codon 47 and one displays a mutation at codon 96 . PZA-MIC determination for these strains revealed slightly elevated values for the strains carrying the mutation at codon $47(25.0 \mu \mathrm{g} / \mathrm{ml})$ compared to the H37Rv control. In a recent study it has been shown that the site of the mutation is leading to varying efficiencies of the mutated pyrazinamidase mediating a wide range of resistance levels from low to high [44]. As the mutation at codon 47 has previously been described by Juréen and co-workers [42] in PZA resistant strains, further investigations are necessary to determine if additional mutations in other parts of the genome might be responsible for the observed low-level resistance in the strains analyzed in this study. Out of all PZA resistant strains three carried the pncA wild type sequence. This indicates that further mutations in as yet unidentified genes are also important for mediating PZA resistance.

\section{Conclusions}

Although resistance mechanisms to INH and RIF are well understood, unknown resistance determining regions and resistance mediating mechanisms appear to play an important role for SM, EMB and PZA, where we observed a relatively low sensitivity for detection of resistance by analysis of common genes. Therefore, it is essential to gather information on further mechanisms leading to drug resistant MTBC strains. For the design 
and implementation of molecular resistance assays it is fundamental to consider strain diversity with respect to resistance mutations in a given geographical setting. Finally, it should be noted that not all variations in well described resistance genes are related to the development of high-level resistance, a finding arguing for a very careful interpretation of molecular resistance assays.

\section{Additional files}

Additional file 1 PCR primers and conditions used for amplification and sequencing. In this table a summary of all primers, including oligonucleotide sequences, used in this study for both DNA amplification and sequencing is given.

Additional file 2 Summary of all sequencing, DST, MIC and genotyping data. This table summarizes all data generated in this study. It comprises sequencing, DST (drug susceptibility testing) and MIC (minimal inhibitory concentration) testing results as well as all genotyping data.

\section{Competing interests}

The authors declare that they have no competing interests.

\section{Acknowledgments}

We thank I. Razio, P. Vock, T. Ubben and L. Dost, Borstel, Germany, for excellent technical assistance.

Parts of this work have been supported by the European Union TM-REST (FP7-202145) and the TB-PAN-NET (FP7-223681) projects.

\section{Author details}

${ }^{1}$ Molecular Mycobacteriology, Research Center Borstel, Borstel, Germany. ${ }^{2}$ German Leprosy and TB Relief Association, Würzburg, Germany. ${ }^{3}$ National Leprosy/TB Reference Laboratory, Freetown, Sierra Leone. ${ }^{4}$ Manager of the National Leprosy and Tuberculosis Programme (NLTP), Ministry of Health and Sanitation, Freetown, Sierra Leone. ${ }^{5}$ National Reference Center for Mycobacteria, Research Center Borstel, Borstel, Germany.

\section{Authors' contributions}

SF: Conception and design of the study, acquisition, analysis and interpretation of data, drafting and revising of the article, given final approval to this version to be published. BO: Conception and design of the study, revising of the article, given final approval to this version to be published. AGG: Conception and design of the study, revising of the article, given final approval to this version to be published. FD: Conception and design of the study, revising of the article, given final approval to this version to be published. ER: Conception and design of the study, interpretation of data, revising of the article, given final approval to this version to be published. SR-G: Conception and design of the study, interpretation of data, revising of the article, given final approval to this version to be published. SN: Conception and design of the study, interpretation of data, drafting and revising of the article, given final approval to this version to be published. All authors read and approved the final manuscript.

Received: 11 January 2012 Accepted: 30 May 2012

Published: 30 May 2012

\section{References}

1. WHO: Report on Global Tuberculosis Control; 2010. www.who.int/tb/ publications/global_report/2010/en/.

2. Yew W-W: Management of multidrug-resistant tuberculosis and extensively drug-resistant tuberculosis: current status and future prospects. Kekkaku 2011, 86:9-16.

3. Corbett EL, Marston B, Churchyard GJ, De Cock KM: Tuberculosis in subSaharan Africa: opportunities, challenges, and change in the era of antiretroviral treatment. Lancet 2006, 367:926-937.

4. WHO: TB country profile Sierra Leone: surveillance and epidemiology; 2009. www.afro.who.int.
5. Bang D, Bengård Andersen A, Thomsen VØ: Rapid genotypic detection of rifampin- and isoniazid-resistant Mycobacterium tuberculosis directly in clinical specimens. J Clin Microbiol 2006, 44:2605-2608.

6. Hillemann D, Rüsch-Gerdes S, Richter E: Evaluation of the GenoType MTBDRplus assay for rifampin and isoniazid susceptibility testing of Mycobacterium tuberculosis strains and clinical specimens. J Clin Microbiol 2007, 45:2635-2640.

7. Zhang Y, Heym B, Allen B, Young D, Cole S: The catalase-peroxidase gene and isoniazid resistance of Mycobacterium tuberculosis. Nature 1992, 358:591-593.

8. Banerjee A, Dubnau E, Quemard A, Balasubramanian V, Um KS, Wilson T, Collins D, de Lisle G, Jacobs WR Jr: inhA, a gene encoding a target for isoniazid and ethionamide in Mycobacterium tuberculosis. Science 1994 263:227-230.

9. Wilson TM, Collins DM: ahpC, a gene involved in isoniazid resistance of the Mycobacterium tuberculosis complex. Mol Microbiol 1996, 19:10251034.

10. Kelley CL, Rouse DA, Morris SL: Analysis of ahpC gene mutations in isoniazid-resistant clinical isolates of Mycobacterium tuberculosis. Antimicrob Agents Chemother 1997, 41:2057-2058.

11. Telenti A, Imboden P, Marchesi F, Lowrie D, Cole S, Colston MJ, Matter L, Schopfer K, Bodmer T: Detection of rifampicin-resistance mutations in Mycobacterium tuberculosis. Lancet 1993, 341:647-650.

12. Finken $M$, Kirschner $P$, Meier $A$, Wrede A, Böttger EC: Molecular basis of streptomycin resistance in Mycobacterium tuberculosis: alterations of the ribosomal protein $\mathrm{S} 12$ gene and point mutations within a functional 16 S ribosomal RNA pseudoknot. Mol Microbiol 1993, 9:1239-1246.

13. Okamoto S, Tamaru A, Nakajima C, Nishimura K, Tanaka Y, Tokuyama S, Suzuki $Y$, Ochi K: Loss of a conserved 7-methylguanosine modification in $16 \mathrm{~S}$ rRNA confers low-level streptomycin resistance in bacteria. $\mathrm{Mol}$ Microbiol 2007, 63:1096-1106.

14. Plinke C, Rüsch-Gerdes S, Niemann S: Significance of mutations in embB codon 306 for prediction of ethambutol resistance in clinical Mycobacterium tuberculosis isolates. Antimicrob Agents Chemother 2006, 50:1900-1902.

15. Ramaswamy SV, Amin AG, Göksel S, Stager CE, Dou SJ, El Sahly H, Moghazeh SL, Kreiswirth BN, Musser JM: Molecular genetic analysis of nucleotide polymorphisms associated with ethambutol resistance in human isolates of Mycobacterium tuberculosis. Antimicrob Agents Chemother 2000, 44:326-336.

16. Plinke C, Cox HS, Zarkua N, Karimovich HA, Braker K, Diel R, Rüsch-Gerdes S, Feuerriegel $S$, Niemann $S$ : embCAB sequence variation among ethambutol-resistant Mycobacterium tuberculosis isolates without embB306 mutation. J Antimicrob Chemother 2010, 65:1359-1367.

17. Jadaun GPS, Das R, Upadhyay P, Chauhan DS, Sharma VD, Katoch VM: Role of embCAB gene mutations in ethambutol resistance in Mycobacterium tuberculosis isolates from India. Int J Antimicrob Agents 2009, 33:483-486.

18. Scorpio A, Zhang $Y$ : Mutations in pncA, a gene encoding pyrazinamidase/ nicotinamidase, cause resistance to the antituberculous drug pyrazinamide in tubercle bacillus. Nat Med 1996, 2:662-667.

19. Dobner P, Bretzel G, Rüsch-Gerdes S, Feldmann K, Rifai M, Löscher T, Rinder $\mathrm{H}$ : Geographic variation of the predictive values of genomic mutations associated with streptomycin resistance in Mycobacterium tuberculosis. Mol Cell Probes 1997, 11:123-126.

20. Ahmad S, Araj GF, Akbar PK, Fares E, Chugh TD, Mustafa AS: Characterization of rpoB mutations in rifampin-resistant Mycobacterium tuberculosis isolates from the Middle East. Diagn Microbiol Infect Dis 2000, 38:227-232.

21. Homolka S, Post E, Oberhauser B, George AG, Westman L, Dafae F, RüschGerdes S, Niemann S: High genetic diversity among Mycobacterium tuberculosis complex strains from Sierra Leone. BMC Microbio/ 2008, 8:103

22. van Soolingen D, Hermans PW, de Haas PE, Soll DR, van Embden JD: Occurrence and stability of insertion sequences in Mycobacterium tuberculosis complex strains: evaluation of an insertion sequencedependent DNA polymorphism as a tool in the epidemiology of tuberculosis. J Clin Microbiol 1991, 29:2578-2586.

23. Sreevatsan S, Pan X, Stockbauer KE, Connell ND, Kreiswirth BN, Whittam TS, Musser JM: Restricted structural gene polymorphism in the Mycobacterium tuberculosis complex indicates evolutionarily recent global dissemination. Proc Natl Acad Sci.USA 1997, 94:9869-9874. 
24. Guo H, Seet $Q$, Denkin S, Parsons $L$, Zhang Y: Molecular characterization of isoniazid-resistant clinical isolates of Mycobacterium tuberculosis from the USA. J Med Microbiol 2006, 55:1527-1531.

25. Kiepiela P, Bishop KS, Smith AN, Roux L, York DF: Genomic mutations in the katG, inhA and aphC genes are useful for the prediction of isoniazid resistance in Mycobacterium tuberculosis isolates from Kwazulu Natal, South Africa. Tuber Lung Dis 2000, 80:47-56

26. Richardson ET, Lin S-YG, Pinsky BA, Desmond E, Banaei N: First documentation of isoniazid reversion in Mycobacterium tuberculosis. Int J Tuberc Lung Dis 2009, 13:1347-1354.

27. Bolotin S, Alexander DC, Chedore P, Drews SJ, Jamieson F: Molecular characterization of drug-resistant Mycobacterium tuberculosis isolates from Ontario, Canada. J Antimicrob Chemother 2009, 64:263-266.

28. Van Rie A, Warren R, Mshanga I, Jordaan AM, van der Spuy GD, Richardson M, Simpson J, Gie RP, Enarson DA, Beyers N, van Helden PD, Victor TC: Analysis for a limited number of gene codons can predict drug resistance of Mycobacterium tuberculosis in a high-incidence community. J Clin Microbiol 2001, 39:636-641.

29. Hauck Y, Fabre M, Vergnaud G, Soler C, Pourcel C: Comparison of two commercial assays for the characterization of rpoB mutations in Mycobacterium tuberculosis and description of new mutations conferring weak resistance to rifampicin. J Antimicrob Chemother 2009, 64:259-262.

30. Zaczek A, Brzostek A, Augustynowicz-Kopec E, Zwolska Z, Dziadek J: Genetic evaluation of relationship between mutations in rpoB and resistance of Mycobacterium tuberculosis to rifampin. BMC Microbiol 2009, 9:10.

31. Van Deun A, Barrera L, Bastian I, Fattorini L, Hoffmann H, Kam KM, Rigouts L, Rüsch-Gerdes S, Wright A: Mycobacterium tuberculosis strains with highly discordant rifampin susceptibility test results. J Clin Microbiol 2009, 47:3501-3506.

32. van Ingen J, Aarnoutse R, de Vries G, Boeree MJ, van Soolingen D: Lowlevel rifampicin-resistant Mycobacterium tuberculosis strains raise a new therapeutic challenge. Int J Tuberc Lung Dis 2011, 15:990-992.

33. Bwanga F, Hoffner S, Haile M, Joloba ML: Direct susceptibility testing for multi drug resistant tuberculosis: a meta-analysis. BMC Infect Dis 2009, 9:67.

34. Mokrousov I, Bhanu NV, Suffys PN, Kadival GV, Yap S-F, Cho S-N, Jordaan AM, Narvskaya O, Singh UB, Gomes HM, Lee H, Kulkarni SP, Lim K-C, Khan BK, van Soolingen D, Victor TC, Schouls LM: Multicenter evaluation of reverse line blot assay for detection of drug resistance in Mycobacterium tuberculosis clinical isolates. J Microbiol Methods 2004, 57:323-335.

35. Spies FS, Ribeiro AW, Ramos DF, Ribeiro MO, Martin A, Palomino JC, Rossetti MLR, da Silva PEA, Zaha A: Streptomycin Resistance and Lineage-Specific Polymorphisms in Mycobacterium tuberculosis gidB Gene. J Clin Microbiol 2011, 49:2625-2630.

36. Ali A, Hasan Z, Moatter T, Tanveer M, Hasan R: M. tuberculosis Central Asian Strain 1 MDR isolates have more mutations in rpoB and katG genes compared with other genotypes. Scand J Infect Dis 2009, 41:37-44.

37. Hillemann D, Kubica T, Rüsch-Gerdes S, Niemann S: Disequilibrium in distribution of resistance mutations among Mycobacterium tuberculosis Beijing and non-Beijing strains isolated from patients in Germany. Antimicrob Agents Chemother 2005, 49:1229-1231.

38. Lipin MY, Stepanshina VN, Shemyakin IG, Shinnick TM: Association of specific mutations in katG, rpoB, rpsL and rrs genes with spoligotypes of multidrug-resistant Mycobacterium tuberculosis isolates in Russia. Clin Microbiol Infect 2007, 13:620-626.

39. Plinke C, Cox HS, Kalon S, Doshetov D, Rüsch-Gerdes S, Niemann S: Tuberculosis ethambutol resistance: concordance between phenotypic and genotypic test results. Tuberculosis (Edinb) 2009, 89:448-452.

40. Ahmad S, Mokaddas E, Jaber A-A: Rapid detection of ethambutol-resistant Mycobacterium tuberculosis strains by PCR-RFLP targeting embB codons 306 and 497 and iniA codon 501 mutations. Mol Cell Probes 2004, 18:299306.

41. Safi H, Fleischmann RD, Peterson SN, Jones MB, Jarrahi B, Alland D: Allelic exchange and mutant selection demonstrate that common clinical embCAB gene mutations only modestly increase resistance to ethambutol in Mycobacterium tuberculosis. Antimicrob Agents Chemother 2010, 54:103-108
42. Juréen P, Werngren J, Toro J-C, Hoffner S: Pyrazinamide resistance and pncA gene mutations in Mycobacterium tuberculosis. Antimicrob Agents Chemother 2008, 52:1852-1854

43. Mphahlele $M$, Syre $H$, Valvatne $H$, Stavrum R, Mannsåker T, Muthivhi T, Weyer K, Fourie PB, Grewal HMS: Pyrazinamide resistance among South African multidrug-resistant Mycobacterium tuberculosis isolates. J Clin Microbiol 2008, 46:3459-3464.

44. Sheen P, Ferrer P, Gilman RH, López-Llano J, Fuentes P, Valencia E, Zimic MJ: Effect of pyrazinamidase activity on pyrazinamide resistance in Mycobacterium tuberculosis. Tuberculosis (Edinb) 2009, 89:109-113.

doi:10.1186/1471-2180-12-90

Cite this article as: Feuerriegel et al:: Sequence analysis for detection of first-line drug resistance in Mycobacterium tuberculosis strains from a high-incidence setting. BMC Microbiology 2012 12:90.

\section{Submit your next manuscript to BioMed Central and take full advantage of:}

- Convenient online submission

- Thorough peer review

- No space constraints or color figure charges

- Immediate publication on acceptance

- Inclusion in PubMed, CAS, Scopus and Google Scholar

- Research which is freely available for redistribution 